\title{
CONHECIMENTO DOS TRABALHADORES DE UMA UNIVERSIDADE PRIVADA SOBRE A PREVENÇÃO DO CÂNCER DE PRÓSTATA
}

\author{
Ana Cláudia Feitosa Lima ${ }^{1}$, Kátia Vanessa Menezes da Silva² ${ }^{2}$ Joselany Afio Caetano ${ }^{3}$, Maria Alzete de Lima ${ }^{4}$, Luciene
} Miranda de Andrade

\begin{abstract}
RESUMO: Estudo descritivo, com o objetivo de investigar o conhecimento dos trabalhadores de uma Universidade privada, no município de Fortaleza - Ceará - Brasil, sobre a prevenção do câncer de próstata. Participaram do estudo 70 trabalhadores do sexo masculino, não docentes, com idade acima de 40 anos. Os dados foram coletados a partir de uma entrevista estruturada, com as seguintes variáveis: dados sociodemográficos; questões sobre o câncer de próstata e exames de prevenção; quem forneceu informações sobre a doença. Os achados revelaram que os entrevistados têm pouco ou nenhum conhecimento sobre o câncer de próstata, não realizam exames preventivos e os que realizaram o fizeram há um ano. O método de prevenção utilizado é o Antígeno Prostático Específico (PSA). Podemos perceber que, embora os homens demonstrem interesse pela temática, poucos pretendem realizar os exames preventivos, o que contribui para o aumento de novos casos de câncer de próstata sem um diagnóstico precoce.
\end{abstract}

PALAVRAS-CHAVE: Neoplasias prostáticas; Prevenção; Conhecimento.

\section{KNOWLEDGE OF WORKERS FROMA PRIVATE UNIVERSITY ABOUT PROSTATE CANCER PREVENTION}

\begin{abstract}
This descriptive study aimed to investigate the knowledge about prostate cancer prevention among workers from a private University in Fortaleza - Ceará - Brazil. Study participants were 70 male workers, non-faculty members, aged over 40 . Data were collected based on a structured interview, with the following variables: socio-demographic data; questions about the prostate cancer and prevention tests; who provided information about the disease. The findings revealed that the interviewees have little or no knowledge about prostate cancer, did not undergo preventive tests and those who did, had taken them one year ago. Prostati-Specific Antigen (PSA) is used as a preventive method. It could be perceived that, although the men demonstrate interest in the theme, few of them intend to undergo preventive tests, which contributes to the increase of new prostate cancer cases without an early diagnosis.
\end{abstract}

KEYWORDS: Prostatic neoplasms; Prevention; Knowledge.

\section{CONOCIMIENTOS DE LOS TRABAJADORES DE UNA UNIVERSIDAD PRIVADA SOBRE LA PREVENCIÓN DEL CÂNCER DE PRÓSTATA}

RESUMEN: Estudio descriptivo, con objeto de investigar el conocimiento de los trabajadores de una Universidad privada, en el municipio de Fortaleza - Ceará - Brasil, sobre la prevención del cáncer de próstata. Participaron del estudio 70 trabajadores del sexo masculino, no docentes, con edad arriba de 40 años. Los datos fueron recolectados por medio de entrevista estructurada, con las siguientes variables: datos sociodemográficos; cuestiones acerca del cáncer de próstata y exámenes de prevención; quién forneció informaciones sobre la enfermedad. Los hallazgos revelaron que los entrevistados poseen poco o ningún conocimiento acerca del cáncer de próstata, no realizan exámenes preventivos y aquellos que los realizaron lo hicieron desde hace un año. El método de prevención utilizado es el Antígeno Prostático Específico (PSA). Se puede percibir que, aunque los hombres demuestren interés por la temática, pocos pretenden realizar los exámenes preventivos, lo que contribuye para el aumento de nuevos casos de cáncer de próstata sin un diagnóstico precoz.

PALABRAS CLAVE: Neoplasias prostáticas; Prevención; Conocimiento.

\footnotetext{
${ }^{1}$ Enfermeira. Mestre em Enfermagem. Docente da Universidade de Fortaleza - UNIFOR.

${ }^{2}$ Enfermeira do Programa de Saúde da Família do Município de Iguatú/CE.

${ }^{3}$ Enfermeira. Doutora em Enfermagem. Docente da Faculdade Federal do Ceará- UFC.

${ }^{4}$ Acadêmica. Curso de Graduação em Enfermagem. Bolsista de iniciação científica da FUNCAP.
}

Autor correspondente:

Ana Cláudia Feitosa Lima

Rua Governador Parsifal Barroso, 400, apart. 1101 - 60355630 - Fortaleza-CE_ Recebido: 18/09/07

E-mail: anaclaudiafl@unifor.br 


\section{INTRODUÇÃO}

Dentre as enfermidades existentes, o câncer constitui uma preocupação crescente da população, pois, embora seja uma doença conhecida há muitos séculos, somente nas últimas décadas tal enfermidade vem ganhando uma dimensão maior, convertendo-se em um evidente problema de saúde pública mundial. No Brasil, estudos revelam que, a partir da década de 1960, as enfermidades infecciosas e parasitárias deixaram de ser a principal causa de morte, dando lugar às doenças do aparelho circulatório e aos processos neoplásicos ${ }^{(1)}$.

No Estado do Ceará, registros do Sistema de Informações de Mortalidade (SIM) da Secretaria Estadual de Saúde revelam que, do total de óbitos ocorridos, um percentual de $15,25 \%$ foram ocasionados por neoplasia, em 2006. Em 2002, o Estado do Ceará ocupava o nono lugar por óbitos no Brasil, sendo o terceiro na região Nordeste, cujos números correspondem a 14,8\% dos óbitos por neoplasias malignas da próstata ${ }^{(2)}$.

Outros dados sugerem que esta doença ocupa a maior taxa de mortalidade e também a segunda em incidência entre homens, com 24,94 casos para cada $100 \mathrm{mil} /$ habitantes. Devido ao aumento significativo das neoplasias, o Ministério da Saúde propôs a Política Nacional de Controle do Câncer, com o intuito de reduzir a incidência e a mortalidade, através da conscientização dos fatores de risco e medidas para a detecção precoce dos cânceres passíveis de rastreamento, com acesso a um tratamento eqüitativo e de qualidade. Em 2001 foi implementada a lei 10.289, de 20 de setembro de 2001, que instituiu o Programa Nacional de Controle do Câncer de Próstata ${ }^{(1)}$.

De acordo com o Instituto Nacional do Câncer, o segundo tipo mais comum entre a população masculina é o câncer de próstata, ocupando o quinto lugar no mundo ${ }^{(3)}$. A próstata é uma parte do sistema reprodutor masculino, do tamanho de uma noz, constituída de tecido glandular e muscular. Sua função é a produção de líquido seminal (esperma) responsável pelo transporte dos espermatozóides que são produzidos nos testículos. Ela também elimina uma substância que não é produzida por nenhum outro órgão e que é possível ser dosada na circulação, cuja denominação é o Antígeno Prostático Específico, mais conhecido como PSA. Em relação ao câncer de próstata, este ocorre quando se localizam células neoplásicas dentro do órgão ${ }^{(4)}$.

Dentre os fatores de riscos, relacionam-se: idade como um marcador importante, uma vez que tanto a incidência como a mortalidade aumentam após os 50 anos; a história familiar pode constituir um aumento no risco de 3 a 10 vezes em relação às características herdadas; estilos de vida compartilhados entre os membros da família; a influência de dieta rica em gordura animal; exposição às substâncias químicas tóxicas como cádmio, benzeno, nitrito de acrílico, dentre outras; os vícios como o tabagismo e o etilismo ${ }^{(5)}$.

Estes fatores de risco associados ao retardo do diagnóstico podem constituir a principal causa do aumento do número de casos de câncer de próstata. Acreditamos que um dos fatores responsáveis pelo diagnóstico tardio da doença se deve à falta de informação da população; ao preconceito a respeito do exame preventivo que é realizado pelo toque retal; à inexistência de procedimentos específicos e sensíveis que possam detectar o tumor em fase microscópica e à dificuldade de implantação de rotinas abrangentes, programadas nos serviços de saúde pública e privadas, que favoreçam a detecção do câncer de próstata.

A identificação nos estágios iniciais das doenças crônicas pode reduzir as taxas de morbidade e mortalidade $^{\left({ }^{()}\right.}$, daí a importância de implementar medidas de prevenção. Neste sentido, despertou-nos o interesse para realização deste estudo, motivados, ainda, pelas observações cotidianas durante a prática profissional de enfermagem, em que se percebe uma maior discussão acerca da saúde da mulher em todas as suas vertentes, em contraste à saúde masculina que pouco se tem abordado. Outro fator que gerou inquietação diz respeito às campanhas de prevenção do câncer, as quais são enfatizadas de modo explícito, em particular pelos profissionais da área de saúde, que se direcionam, essencialmente, à prevenção contra neoplasias de colo uterino e mamas. Entretanto, a prevenção de enfermidades específica do sexo masculino, como, por exemplo, o processo neoplásico que acomete a próstata, não é percebida nas estratégias e planejamentos de cuidado de saúde.

Vale ressaltar que tivemos grandes dificuldades para encontrar artigos ou outras publicações que tratassem da especificidade estudada nesta pesquisa. Em consulta aos bancos de dados MEDLINE e LILACS, encontrou-se somente três referenciais de trabalhos que tratavam do objeto específico desta pesquisa. A partir dessa perspectiva, objetivou-se investigar o conhecimento dos trabalhadores do sexo masculino de uma universidade privada sobre a prevenção do câncer de próstata.

\section{DESCRIÇÃO METODOLÓGICA}

Estudo descritivo, com abordagem quantitativa, desenvolvido em 2006, com funcionários de uma Universidade privada, localizada na região metropolitana de Fortaleza-CE. Participaram do estudo 
70 trabalhadores do sexo masculino, não docentes, com idade a partir de 40 anos e que aceitaram o convite para participar da pesquisa.

Para coleta de dados foi elaborado um instrumento para direcionar a entrevista, que buscava respostas objetivas e subjetivas, tendo como variáveis: dados de identificação e dados sociodemográficos (faixa etária, procedência, estado civil, cargo ou função) e questões sobre o significado do câncer de próstata; a realização de exames de prevenção; quem forneceu informações sobre a doença; quais exames preventivos realizaram e há quanto tempo foram feitos; além do tipo de material informativo que gostariam de ter acesso. Os dados foram coletados no período de janeiro a março de 2006 e apresentados em forma de tabelas e analisados à luz da literatura.

Este estudo foi aprovado pelo Comitê de Ética em Pesquisa, em cumprimento da Resolução 196/96(6) da Comissão Nacional de Ética em Pesquisa (CONEP) que regulamenta a pesquisa envolvendo seres humanos, pelo processo $\mathrm{n}^{\circ}$ 513/2004. O instrumento de coleta de dados foi acompanhado pelo Termo de Consentimento Livre, que garante a participação voluntária dos entrevistados e o sigilo sobre sua identificação.

\section{ANÁLISE E DISCUSSÃO DOS RESULTADOS}

Os trabalhadores entrevistados abrangem uma faixa etária entre 40 e 60 anos; naturais de Fortaleza, 46 (65,7\%); casados, 46 (65,7\%); solteiros, 16 (22,9\%); separados, 6(8,6\%) e viúvos, 2 (2,8\%). Quanto à ocupação que exerciam na instituição, as principais foram: serviços gerais, escriturário, segurança e jardineiro. É importante destacar algumas características dos sujeitos da pesquisa, pois acreditamos que possam influenciar nos relatos.

Quando investigado sobre conhecimento da doença, os discursos revelam a desinformação da população masculina do estudo sobre o câncer de próstata. A maioria, $26(37,1 \%)$ o conceitua como simplesmente sendo uma doença que atinge os homens; nove $(13,3 \%)$ a definem como uma doença que dá na próstata; sete $(10,0 \%)$ uma doença que mata; cinco $(7,1 \%)$ tumor maligno que se desenvolve no homem; três $(4,3 \%)$ problema que dá no ânus; dois $(2,8 \%)$ comentam: é um câncer no pênis, caroço que nasce nos testículos e calo que se forma na via uretral. Em menor número, 1 (1,4\%) diz: câncer que dá na próstata e que deixa o homem impotente e estéril; degeneração de algum órgão; exame que o idoso faz depois de certa idade e glândula que o homem tem e que incha. Um percentual de 14,3\% (10) respondeu: não sei explicar (Tabela 1).

$\mathrm{O}$ câncer é uma enfermidade que tem como característica um crescimento desordenado (maligno) das células que invadem os tecidos e órgãos e que pode espalhar-se para outras regiões do corpo (metástase). $\mathrm{O}$ número de casos de câncer tem aumentado cada vez mais em todo o mundo. Um dos aspectos a ser destacado é que, à medida que a população envelhece, a probabilidade de aumento da incidência e prevalência cresce consideravelmente. Outro ponto é a suscetibilidade aos fatores de risco como, antecedentes familiares, idade, raça, dieta rica em gordura animal, radicais ionizantes e fatores ambientais ${ }^{(1)}$.

Observamos que existem muitos conceitos errôneos por parte dos sujeitos dessa pesquisa, mas percebemos interesse desses homens em conhecer a respeito do câncer de próstata. Assim, acreditamos que, como profissionais de saúde, devemos desenvolver atividades educativas, visando a um maior esclarecimento sobre a doença e, principalmente, sobre as medidas preventivas, pois essa desinformação é preocupante, já que o conhecimento sobre a doença e os meios de prevenila podem ser decisivos sobre sua evolução.

Tabela 1- Distribuição dos trabalhadores não docentes de uma Universidade privada, segundo conhecimento do significado do câncer de próstata. Fortaleza-CE, 2006

\begin{tabular}{lcc}
\hline Respostas & n & \% \\
\hline Doença que atinge os homens. & 26 & 37,1 \\
Doença que dá na próstata. & 09 & 13,2 \\
Doença que mata. & 07 & 10,0 \\
Tumor maligno que se desenvolve no homem. & 05 & 7,1 \\
Problema que dá no ânus. & 03 & 4,3 \\
É um câncer no pênis. & 02 & 2,8 \\
Caroço que nasce nos testículos. & 02 & 2,8 \\
Calo que se forma na via uretral. & 02 & 2,8 \\
Câncer que dá na próstata e que & & \\
deixa o homem impotente e estéril. & 01 & 1,4 \\
Degeneração de algum órgão. & 01 & 1,4 \\
Exame que o idoso faz depois de certa idade. & 01 & 1,4 \\
Glândula que o homem tem e que incha. & 01 & 1,4 \\
Não sei explicar & 10 & 14,3 \\
\hline Total & $\mathbf{7 0}$ & $\mathbf{1 0 0 , 0}$ \\
\hline
\end{tabular}

A idéia de o câncer de próstata significar causa de morte, assim como a percepção de que este pode levar ao estado de esterilidade e impotência, nos remete 
ao que foi constatado em um estudo anterior sobre cultura, saúde e doença: algumas doenças, entre elas o câncer, permanecem arraigadas no folclore devido à associação a crenças tradicionais sobre a natureza moral da saúde ${ }^{(7)}$. Outro aspecto levantado foi a associação que se faz do câncer prostático à terceira idade, e embora nosso público fosse composto por maiores de 40 anos, e sabendo que a prática de exames de detecção do câncer prostático é prioritária nesse grupo etário, como preconiza o INCA e o Ministério da Saúde, percebemos que estes não estão procurando um profissional de saúde para realizar seus exames.

Para completar a investigação do conhecimento sobre o câncer de próstata, dos 70 homens, 32 disseram que as informações foram adquiridas com profissionais de saúde; 12 (37,5\%) por colegas de trabalho; $11(34,4 \%)$ na televisão; cinco $(15,7 \%)$ em palestras; $2(6,2 \%)$ em folhetos informativos e $(3,1 \%)$ em revistas. Aqueles que não receberam informação sobre o tema em questão somaram $38(54,3 \%)$.

Portanto, destacamos a importância de que campanhas educativas sejam feitas, promovendo ações com os profissionais de saúde, no intuito de fornecer materiais ilustrativos e educativos que facilitem a compreensão e conscientização do público alvo, como forma de promoção da saúde.

Quanto aos exames utilizados para a deteç̧ão precoce do câncer de próstata, $54(77,1 \%)$ referem nunca ter feito, enquanto que $16(22,9 \%)$ tiveram acesso a algum tipo de exame preventivo. Desses, dez $(62,5 \%)$ fizeram o PSA e seis $(37,5 \%)$ o PSA mais toque retal. Com relação à periodicidade na realização desses exames, um total de oito $(50,0 \%)$ havia feito há um ano; sete $(43,8 \%)$ o fizeram há mais de um ano e um $(6,2 \%)$ a menos de seis meses.

$\mathrm{O}$ exame de sangue para dosagem de PSA é considerado um exame extremamente útil para monitorar o aparecimento do câncer, pois quando sua concentração encontra-se elevada, uma investigação merece ser realizada, bem como a glicoproteína liberada pelas células prostáticas. Tanto as células normais quanto as malignas secretam esta substância. Porém, no câncer, os níveis na circulação sanguínea se elevam. Vale ressaltar que este tipo de exame é apenas um complemento na realização do exame do toque retal, pois cerca de $30 \%$ dos pacientes com tumor maligno da próstata, em seu estado inicial, podem ter o PSA normal, sem sintomatologia alguma, visto que o câncer é uma doença de início insidioso. Apesar disto, em 30\%, geralmente, o nódulo já é palpável ao toque retal. Por isso, é importante que a partir dos 40 anos o homem anualmente deva se preocupar em ter sua próstata examinada, através do toque retal e $\mathrm{PSA}^{(3)}$.

O exame de toque retal é uma medida preventiva de baixo custo. No entanto, o medo de ser tocado e de sentir dor, ou eventual ereção que possa ser confundida com prazer e o constrangimento pelo fato, são alguns fatores que impedem o homem a procurar os serviços de saúde, associados muitas vezes ao despreparo dos profissionais de saúde em abordar os aspectos simbólicos que permeiam o imaginário masculino para realização deste exame ${ }^{(7)}$.

Assim, o câncer prostático e seu rastreamento precoce ainda representam um assunto pouco discutido com a população masculina, ficando os homens alheios a este sério problema de saúde pública no Brasil. Isto pode acarretar um impacto negativo na saúde de pessoas do nosso País, uma vez que, desconhecendo a gravidade do problema, poucos homens hão de procurar os serviços de saúde ${ }^{(8)}$.

A informação é fundamental para a detecção do câncer de próstata. Acreditamos que havendo informações sobre o câncer, mesmo simples, podem ser valiosas, principalmente sobre o rastreamento e a importância da prática de exames de detecção, os homens se mostrarão mais sensibilizados e poderão pensar melhor suas atitudes, crenças e comportamento diante da prática destes exames ${ }^{(9)}$.

Como profissionais comprometidos com a promoção da saúde da população, devemos estender nossas ações na atenção primária à saúde, estar atentos e verificar se os usuários se encontram na idade de iniciar os exames de detecção e outras doenças associadas, como também incentivar e sensibilizá-los quanto à importância dos homens aprenderem acerca da manutenção da saúde como um todo.

Sobre as sugestões dadas pelos trabalhadores para o incentivo à realização do exame de prevenção do câncer de próstata, $20(28,6 \%)$ enfatizaram as campanhas educativas; $17(24,2 \%)$ sugeriram que os homens procurem um médico; 16 (22,9\%) abordaram a necessidade de acabar com o preconceito, para incentivar os homens a procurarem esclarecimentos sobre a doença e as formas de prevenção; $16(22,9 \%)$ sugeriram que todo homem deveria ter consciência e cuidar de sua saúde e um $(1,4 \%)$ refere que os serviços só lembram das mulheres e os homens são esquecidos (Tabela 2). 
Tabela 2 - Distribuição dos trabalhadores não docentes de uma Universidade privada em relação à conscientização sobre a realização do exame de prevenção do câncer de próstata. Fortaleza-CE, 2006

\begin{tabular}{lcc}
\hline Respostas & n & \% \\
\hline Que ocorressem campanhas educativas, que & & \\
fosse mais divulgado. & 20 & 28,6 \\
Que procure um médico. & 17 & 24,2 \\
Precisa acabar o preconceito. & 16 & 22,9 \\
Todo homem deveria ter consciência & & \\
e cuidar da saúde. & 16 & 22,9 \\
Só lembram das mulheres. & & \\
Os homens são esquecidos. & 1 & 1,4 \\
\hline Total & $\mathbf{7 0}$ & $\mathbf{1 0 0 , 0}$ \\
\hline
\end{tabular}

Diante do exposto, vimos que a falta de informação interfere direta e negativamente na detecção do câncer prostático, não apenas em termos de conhecimento sobre a doença em si e seu rastreamento, mas também em termos do acesso aos serviços de saúde ${ }^{(9)}$. Por isso, torna-se necessário que os serviços de saúde estejam preparados para absorver este tipo de clientela, adequado-se ao perfil tão diversificado desse público.

O conhecimento da patologia e o acesso aos serviços preventivos e de diagnósticos são considerados pontos-chaves na prática preventiva. Conhecendo-se a evolução do câncer de próstata e dos métodos de diagnóstico precoce, e dispondo-se de condições de acesso aos serviços médicolaboratoriais, potencialmente o câncer de próstata pode ser detectado numa fase inicial e com isto o caso apresentar, na maioria das vezes, melhor prognóstico ${ }^{(9)}$.

\section{CONSIDERAÇÕES FINAIS}

Os resultados evidenciaram que os homens do estudo têm pouco conhecimento sobre o câncer de próstata. Poucos realizaram o exame de prevenção, mas acreditam que deve haver estratégias de incentivo às campanhas educativas, uma maior assistência médica e acabar com o preconceito em torno da doença. Isso contribuiria para maior adesão à realização de exames preventivos.

Este trabalho teve como objetivo principal investigar o conhecimento dos trabalhadores de uma universidade privada sobre a prevenção do câncer de próstata, em que pode concluir que eles não estão bem esclarecidos com relação ao significado do câncer de próstata, quais os exames preventivos, e principalmente sua importância. Outro ponto importante trata-se da carência de materiais didáticos e informativos para que os trabalhadores possam ser esclarecidos com relação à doença em questão.

É importante deixar claro que este estudo deve trazer uma reflexão sobre a problemática em questão, e também que se pense em espaços para atuação dos profissionais de saúde, de modo que a clientela masculina possa ser favorecida com ações que abordem sua sexualidade.

Estudos comparativos a serem realizados com este tipo de população, usando metodologia semelhante, poderiam contribuir para confirmar ou reforçar algumas afirmações. Reconhecemos as limitações do nosso estudo e esperamos que este seja somente um, dentre muitos outros que deverão ser desenvolvidos para um melhor e maior acompanhamento do câncer de próstata. Devido à escassez na literatura e aos resultados deste estudo, acreditamos que são preocupantes e dignos de maior aprofundamento os fatores comentados neste trabalho.

Outro aspecto relevante é a importância que uma instituição deve dar à saúde do trabalhador, e que ações de educação em saúde devem ser implementadas, assim como uma assistência médica estendida de maneira mais holística à saúde do homem.

\section{REFERÊNCIAS}

1. Brasil, MS. Ações de enfermagem para o controle do câncer de próstata: uma proposta de integração ensinoserviço. Rio de Janeiro: INCA; 2002.

2. Ceará SS. Coordenadoria de Políticas em Saúde. Núcleo de Epidemiologia. Fortaleza: Novembro de 2003. [Acesso em 2004 Out 14] Disponível em: http://www. sesa.ce.gov.br.

3. Brasil, MS. Secretaria Nacional de Assistência à Saúde. Instituto Nacional do Câncer. Programa de Controle do Câncer de Próstata: Documento de consenso. Rio de Janeiro: INCA; 2002.

4. Mendes ADB. Exame de toque retal: é preciso acabar com o preconceito. São Paulo: Novembro de 2004. Disponível em: http://www.iov.com.br/biblioteca.

5. Tucunda LTCM, Sá VHLC, KoshimuraET, Prudente FVB, Santos ESTS, Samano EST, et al. Estudo da atitude e do conhecimento dos médicos não oncologistas em relação às medidas de prevenção e rastreamento do câncer. Rev 
Assoc Med Bras. 2004 Jul/Set;50(3):257-62.

6. Ministério da Saúde. (BR). Conselho Nacional de Saúde. Diretrizes e normas regulamentadoras de pesquisa envolvendo seres humanos. Resolução n 196 de 10 de outubro de 1996. Brasília; 1996.

7. Gomes, C. Doenças cancerosas. São Paulo: Junho de 2004. [Acesso em 2004 Ago 16]. Disponível em: http:// www.cancerdeprostata/contribuições.

8. Pereira JRS, Lima CAS, Albuquerque IMN, Graça JRV. Conhecimento de mototaxistas do município de Sobral sobre detecção do câncer de próstata. Rev Scientia Mater. 2003 Jun;2(1):79-93.

9. Miranda PSC, Côrtes MCJW, Martins ME, Chaves PC, Santarosa RC. Práticas de diagnóstico precoce de câncer de próstata entre professores da faculdade de medicina - UFMG. Rev Assoc Med Bras. 2004 Jul/ Set;50(3):272-5. 